\title{
Working with MAPPA: ethics and pragmatics
}

\author{
Richard Taylor \& Jessica Yakeley
}

\begin{abstract}
SUMMARY
Multi-agency public protection arrangements (MAPPA) have been in operation for around 18 years in England and Wales. The primary purpose is for the sharing of information between agencies regarding the risk management of offenders returning to the community from custodial and hospital settings. The legal framework regarding information by psychiatrists is not dealt with in one single policy or guidance document. Psychiatrists must use their clinical and professional judgement when engaging with the MAPPA process, mindful of guidance available from professional bodies such as the Royal College of Psychiatrists, General Medical Council and British Medical Association.
\end{abstract}

\section{LEARNING OBJECTIVES}

After reading this article you will be able to:

- Learn the legal and political background that led to the formation of MAPPA

- Understand the structure and function of MAPPA

- Understand the role of psychiatrists in the MAPPA process

\section{DECLARATION OF INTEREST}

R.T. is a member of the London Strategic Management Board for MAPPA.

Multi-agency public protection arrangements (MAPPA) were introduced in England and Wales in 2000 by the then Labour government with the aim of minimising the risk of sexual and violent offences posed by identified high-risk individuals living in the community. In 2000, the Police, Probation and Prison Services were established as the 'responsible authority' (RA) to oversee statutory arrangements for public protection by the identification of high-risk offenders, the assessment and management of their risk, and the sharing of relevant information among the agencies involved. Legislation established MAPPA as a set of provisions to guide inter-agency cooperation, rather than as a new body, and these provisions did not come with significant extra resources.

Although MAPPA-related issues most commonly occur in forensic settings, psychiatric patients who are eligible for MAPPA may be found in all areas of mental health. In this article we will review the social, political and legislative changes that led to the formation and subsequent development of MAPPA, provide practical advice for psychiatrists on how to refer and work effectively with MAPPA, and explore some of the legal and ethical issues involved in medical confidentiality, information sharing and disclosure. More comprehensive guidance for working with MAPPA is provided by the Royal College of Psychiatrists' Faculty of Forensic Psychiatry (Taylor 2013).

\section{Historical background}

\section{Public protection in the USA - Megan's Law}

The inception of MAPPA in 2000 grew out of increasing international concern about the risk to the public from sex offenders over the previous decades. In the USA, public outrage arising from high-profile cases of sexual offending against children led to demands that information about paedophiles should be openly available to the public. In California, sex offenders convicted after 1944 have been required, since 1982, to register with the Chief of Police or County Sheriff within 5 days of changing residence (Cal. Penal Code - Title 9 amended by Stats. 1982, Ch. 1111 §2). In 1995, a child molester identity telephone line was set up. However, it was the case of Megan Kanka that led to a fundamental change in the US legal system in the management of sex offenders.

Megan Kanka was a 7-year old child who was raped and murdered in New Jersey in 1994 by a known paedophile called Jesse Timmendequas, who had recently moved into the residential area where Megan lived. Megan's parents argued that if they had known of the risk Timmendequas had posed, Megan would not have been killed, and they successfully campaigned to change the law to the mandatory community notification of sex offenders so that their criminal history could be known to the public. Megan's Law is the name for a federal law, and the informal name for subsequent state laws, in the USA requiring law enforcement authorities to make information available to the public regarding registered sex offenders. Federal Megan's Law was enacted as a subsection of the Jacob Wetterling Crimes Against Children and
Richard Taylor is a consultant forensic psychiatrist at the Fixated Threat Assessment Centre in North London Forensic Service. Jessica Yakeley is a consultant psychiatrist in forensic psychotherapy at Tavistock and Portman NHS Trust. Correspondence Richard Taylor, North London Forensic Service, Camlet 3, Chase Farm Hospital, Enfield, Middlesex EN2 8JL, UK. Email: richard.taylor18@nhs.net

\section{Copyright and usage} (C) The Royal College of Psychiatrists 2019 
Sexually Violent Offender Registration Act of 1994. Many American states now list sex offenders on the internet and information about a suspected offender can be ascertained by entering their name or ZIP code. In Louisiana, personal email alerts can be set up by any member of the public to receive a message if a paedophile moves into their area. In Oregon, convicted paedophiles are required to place a sign in their window, and in Washington State, sex offenders without a fixed residence must report to the police on a weekly basis.

\section{The UK experience}

In England and Wales MAPPA was introduced after 15 years of a marked harshening of the emotional tone of penal policy that started with the reforms of Conservative home secretary Michael Howard and continued under the subsequent Labour home secretary David Blunkett (Liebling 2012). This shift in a more punitive direction was exploited by the tabloid press, which promulgated exaggerated and often inaccurate accounts of the risk of sex offenders to the public.

Calls for a law similar to Megan's Law came after the much-publicised killing of Sarah Payne, an 8year-old girl, in 2000. The former tabloid News of the World campaigned for laws to protect children from sex offenders. The child sex offender disclosure scheme in England and Wales ('Sarah's Law') was introduced in 2010, and it allows anyone to formally ask the police if someone with access to a child has a record for child sexual offences. Police will reveal details confidentially to the person most able to protect the child (usually parents, carers or guardians) if they think it is in the child's interests.

The national furore following the revelations regarding Jimmy Saville's extensive sexual offences and paedophilic activities over many years, and more recent disclosures about sexual abuse within

\section{BOX 1 Legislative changes regarding the management of high-risk offenders}

Limited parole for sex offenders 1998

1994

1997

1997
Criminal Justice and Public Order Act introduced longer sentences for public protection for certain types of offences

Crime (Sentences) Act introduced a mandatory life sentence for the second serious offence

Sex Offenders Act required that sex offenders register with police on release from prison
Crime and Disorder Act brought in the sex offender order 2000 Criminal Justice and Court Services Act brought in MAPPA

2003 Criminal Justice Act

2003 Sexual Offences Act

2004 Domestic Violence, Crime and Victims Act

2007 Amendments to the Mental Health Act 1983 football and other professional groups, have fuelled public outrage and demands for tougher legislation.

Alongside these issues about sex offenders, concerns arose about the risk posed by those with a personality disorder. This led to the Dangerous Severe Personality Disorder (DSPD) initiative, a controversial programme established in 2001 for the management and treatment in prisons and forensic hospitals of detained offenders assessed as posing significant risk of harm to others and whose risk was linked to their personality disorder. The programme was subsequently disbanded in favour of a reconfigured national strategy for managing high-risk personality disordered offenders (the Offender Personality Disorder Pathway), involving both the criminal justice system and National Health Service (NHS) (Joseph 2012).

There are clearly human rights issues concerning the stigmatisation of offenders and their right to privacy. Following the implementation of Megan's Law there were several documented cases of vigilantism and violence directed against known offenders. Moreover, evidence from the USA showed that if an offender's details were automatically made public, a proportion would no longer comply with the notification requirements and would disappear, leaving the authorities unsure of their whereabouts and unable to monitor them (Fitch 2007).

Where limited or inaccurate information about a suspected offender is disclosed it can quickly spread via social media or adverse press coverage. In the UK, the News of the World's campaign led to widespread public disorder, including an attack on the home of a paediatrician mistaken for a paedophile in Portsmouth. Furthermore, there has been concern following the Jimmy Saville revelations that the media attention on celebrity paedophiles risks diverting attention away from the more widespread child sexual abuse that can occur in children's homes and other institutions, or that which occurs within families.

\section{Legislative changes in England and Wales}

These public, media and political calls for more public protection have shaped an increasingly complex legislative framework for the management of perceived dangerous offenders (Box 1). In the 1980s there was limited parole for sex offenders, but in the 1990s several laws were brought in which introduced longer sentences for public protection for certain types of offences and which required sex offenders to register with police on release from prison. However, it was the Criminal Justice Act 2003 that made the most radical changes to the 
BOX 2 Civil orders of the Sexual Offences Act 2003

Sexual offences prevention orders (SOPOs), aimed at preventing preparatory behaviour by convicted sexual offenders, for example banning the offender from entering a park, taking a recreational walk unless accompanied by another adult, seeking the company of any young person under 16 , accessing the internet, or possessing equipment capable of creating photographs of children.

Notification Orders, which require sexual offenders abroad to register with the police on their return to the UK. Foreign travel orders, which restrict sexual offenders travels abroad.

Risk of sexual harm orders, which restrict the activities of individuals suspected of being involved in grooming children for sexual activity but who have no previous convictions or cautions for sexual offences.

structure of prison and community sentences, introducing indeterminate sentences for public protection (IPPs) and extended sentences for public protection (EPPs). Release from an IPP is at the discretion of the Parole Board on grounds of public safety, and an EPP allows for supervision of offenders in the community for an extended period. In the same year the Sexual Offences Act introduced four civil orders that can be used to protect the public, especially children and other vulnerable adults, from sex offenders (Box 2).

Subsequent legislation has affected the management of mentally disordered offenders. The Domestic Violence, Crime and Victims Act 2004 extended the rights of victims of domestic abuse, including the right of a victim of a mentally disordered offender detained in hospital to receive information about that patient's discharge. Amendments to the Mental Health Act 1983 in 2007 introduced supervised community treatment for patients following detention in hospital, to allow them to live in the community while subject to certain conditions to ensure they continue with medical treatment.

\section{What is MAPPA?}

\section{The responsible authority}

The Criminal Justice and Court Services Act 2000, followed by further provisions of the Criminal Justice Act 2003, established the police, probation and prison services as the 'responsible authority' to oversee statutory arrangements in England and Wales for public protection by the identification of high-risk offenders, the assessment and management of their risk, and the sharing of relevant information among the agencies involved. The responsible authority is charged with establishing MAPPA in each of the 42 criminal justice areas in England and Wales. In each area, strategic management boards (SMBs), with lay advisor members to represent the public interest, regularly review and monitor these arrangements, as well identifying and developing the training of those working in MAPPA and acting as a link with relevant local policy and practice. Accordingly, the implementation of MAPPA varies to some extent in different parts of the country.

\section{The duty to cooperate}

The Criminal Justice Act 2003 introduced a 'duty to cooperate' clause for a range of agencies other than the police, probation and prison services involved in MAPPA. This clause was intended to enhance multi-agency work by coordinating the involvement of these different agencies in assessing and managing risk, and to enable every agency with a legitimate interest to contribute as fully as its existing statutory role and functions require, in a way that complements the work of other agencies. The agencies concerned (dubbed 'duty-to-cooperate' agencies) are listed in section 325(6) of the Criminal Justice Act 2003 and they include local authority youth offending teams, social care services (for children and adults), housing (including registered social landlords), education, Jobcentre Plus, the UK Border Agency and health. 'Health' is listed as the health authority or strategic health authority, the primary care trust or local health board, and the National Health Service (NHS) trust. The responsible authority is likely to deal most frequently with mental health trusts because many MAPPA cases involve offenders with a history of mental disorder.

In practice, this means that representatives from each agency are expected to attend case conferences, share information about offenders and provide advice regarding management. In the case of health, there should ideally be a standing representative as a single point of contact, along with individual team members on a case-by-case basis. It is important to note that MAPPA is a set of provisions, not a body in its own right, and therefore cannot direct any agency do anything that is outside its usual responsibilities. The agencies involved in MAPPA retain their primary responsibilities independently of what they do under MAPPA, but they are expected to prioritise interagency working that will protect the public from serious harm. It is intended that the strength of MAPPA arises from all agencies collaborating and working effectively together. 
The 'duty to cooperate on health' requires that a suitably qualified and senior mental health practitioner assists MAPPA in the risk assessment and management of mentally disordered offenders eligible for MAPPA, and provides case management for those classified at level 2 or level 3 (see below). It is important to note that the duty is imposed on an NHS trust rather than on an individual staff member. MAPPA is not solely the province of forensic mental health services, but of all trusts and other health organisations, which should be aware of their obligations regarding cooperating with MAPPA under the Criminal Justice Act and should have policies that cover the role of psychiatrists and other members of the multidisciplinary team in the MAPPA process.

\section{The MAPPA framework}

\section{Core functions}

The MAPPA framework is made up of four overlapping and complementary core functions, which the responsible authority must ensure are established across the agencies involved. These core functions are:

- the identification of offenders meeting statutory criteria for management under MAPPA (MAPPA offenders)

- the assessment of the risk that these offenders pose

- the management of that risk

- the safe and secure sharing of relevant information among the agencies involved in assessing and managing the risk of MAPPA offenders.

\section{BOX 3 MAPPA level 1}

Mr A, a 45-year-old manager with a diagnosis of bipolar disorder, committed a contact sexual offence against a 15-year-old schoolgirl in a public park while floridly hypomanic and disinhibited. There was no history of sexual or other offending prior to this incident. Although diverted from police custody to a psychiatric intensive care unit, he was later convicted after pleading guilty, as expert evidence regarding a possible insanity defence was equivocal. Although he had recovered from the acute episode, at sentencing he was made subject to a hospital order (under section 37 of the Mental Health Act 1983) on grounds of the nature of his illness, given the need for rehabilitation and risk management, rather than because of active symptoms. He was also placed on the sex offenders register. Within a week of the hearing the in-patient team social worker notified the MAPPA administrator at the public protection unit (PPU or Jigsaw Team) that Mr A was in hospital. At the point of discharge some weeks later on a community treatment order, MAPPA was again notified of his change of status, but he was not referred to MAPPA as the risk of offending was thought to be entirely related to his risk of relapse of bipolar disorder and therefore he could be managed by a single agency - mental health - at level 1 of MAPPA, with no need for referral into level 2 for interagency management

\section{MAPPA categories of offender}

The list of 153 sexual and violent convictions which render the individual eligible for MAPPA are listed in schedule 15 of the Criminal Justice Act 2003. This wide range of offences includes acts of terrorism, murder and manslaughter, kidnap, sexual exploitation, hijacking, arson, prostitution, sexual activity with children and illegal paraphilic disorders.

Offenders who fall within the MAPPA remit are divided into three broad categories specified in section 327 of the Criminal Justice Act:

- category 1: registered sex offenders - offenders required to comply with the notification (registration) requirements set out in the Sexual Offences Act 2003 (the 'sex offenders' register');

- category 2: violent offenders sentenced to imprisonment of 12 months or more, other sex offenders not required to register, and offenders detained under hospital or guardianship orders, either under the Mental Health Act or after being found unfit to plead or not guilty by reason of insanity;

- category 3: other offenders who do not fall into categories 1 or 2 , but because of the severity of their offences are considered to pose a risk of serious harm to the public ('serious harm' is defined as 'harm which is life threatening or traumatic and from which recovery, whether physical or psychological, can be expected to be difficult or impossible'). Category 3 is intentionally broad, and most of the offenders in this category are those who committed serious violent or sexual crimes prior to the introduction of MAPPA legislation.

The majority of MAPPA offenders come from within the prison system, but some have received hospital disposals. Less than $5 \%$ are assessed as posing the highest risk or requiring the most complex inter-agency risk management.

\section{MAPPA levels of management}

There are three tiers or levels to the MAPPA management system at which risk is assessed and managed.

\section{Level 1: Ordinary risk management}

This level is used in cases where the risk posed by the offender can be managed by one lead agency, such as the police or probation service, or where there is another active risk management process in place, such as within the health service. Offenders managed at this level tend to have been classified as low or medium risk (Box 3).

\section{Level 2: Active multi-agency management}

This level is for offenders posing a high or very high risk of serious harm, whose management requires 
the active involvement of more than one agency. The work is coordinated at monthly multi-agency meetings with permanent representation of the core agencies of the police, probation and prison services, supplemented by representatives of other involved agencies, including health, where needed. Level 2 may also be used for offenders assessed at lower risk but who require the active involvement of agencies to manage the risks of serious harm, or for offenders who have been previously managed at level 3 but no longer meet the criteria (Box 4).

\section{Level 3: Active enhanced multi-agency management}

Level 3 is for a small number of cases that meet the criteria for level 2 , but where the management issues require senior representation from the responsible authority and duty-to-cooperate agencies. This may be for offenders who are not assessed as posing high or very high risk of serious harm, but for whom there is a high likelihood of media scrutiny

\section{BOX 4 MAPPA level 2}

$\mathrm{Mr} \mathrm{B}$ was detained in a private sector medium secure forensic unit on a restricted hospital order under sections 37 and 41 of the Mental Health Act 1983 for a serious offence of wounding with intent (section 18 of the Offences Against the Person Act 1861) carried out against his estranged partner. He committed the offence during a relapse of schizophrenia in the context of harmful use of synthetic cannabinoids. Within a week of the sentencing hearing, the local MAPPA team for his home borough was notified of his status, as was the local MAPPA team in the area where the medium secure unit was situated. After an 18-month period during which he had been stabilised on depot antipsychotic and had completed group and individual work, including reasoning and rehabilitation, focused on substance misuse and offending, an application was made to the Ministry of Justice mental health casework section for unescorted leave. Mr B's estranged partner had given birth some months after the offence and his paternity had been established unequivocally. He had instructed a family solicitor to help him negotiate contact with his child on a supervised basis, but his estranged partner -the victim of the index offence - had requested an exclusion zone via the victim liaison officer. At the point of applying for unescorted leave an application for a level 2 inter-agency MAPPA meeting was made to discuss the victim and child protection issues with other MAPPA agencies, so that a care plan and exclusion zone could be agreed before the first unescorted leave. $\mathrm{Mr} \mathrm{B}$ remained a level 2 case (on both home and host MAPPA case-loads) during the discharge planning period. About a year after successful conditional discharge he was downgraded to level 1 after his partner and child permanently moved overseas.

\section{BOX 5 MAPPA level 3}

$\mathrm{Mr} \mathrm{C}$ had committed a homicide in the context of being subjected to significant provocation by his victim in the form of insults and in the context of what was thought to be either paranoid personality disorder or delusional disorder. There was a disagreement between whether a mental health disposal was appropriate or not. At sentencing by the Crown Court he was made subject to a determinate prison sentence. While in prison custody there was further incident of disturbed behaviour and aggression and again there was a disagreement about diagnosis as to whether he had a delusional disorder or a paranoid personality disorder. He remained in prison custody but was very isolated and in long-term segregation. As he approached his automatic release date from his determinate sentence, the family of the victim became aware and launched a campaign in the local press to publicise their concerns. The probation offender manager in the prison made a referral for a level three MAPPA meeting, which was convened by the home catchment area MAPPA although he was in a dispersal prison many miles away. At the level 3 psychiatric experts about diagnosis and
MAPPA meeting various issues were discussed including further psychiatric assessment as there had never been an in-patient assessment of diagnostic issues such as an untreated delusional disorder or possibly schizophrenia. A section 47/49 order (transfer to hospital of sentenced prisoner under the Mental Health Act 1983) was made by The Section. A media strategy was agreed given the local press coverage and the police appointed a family liaison officer to work with the deceased victim's family. After a series of level-three meetings convened at the local police station, and following a further psychiatric review by the catchment area forensic service, he was transferred to hospital a few weeks before sentence expiry. In hospital he was given a diagnosis of delusional disorder and responded to anti-psychotic therapy. After 18 months of further in-patient rehabilitation he was eventually made subject to a Community Treatment Order and discharged to 24-hour supported accommodation an appropriate distance from the victim's relatives and he was later downgraded to level 2 MAPPA. Ministry of Justice Mental Health Casework

or public interest and therefore a need to ensure that public confidence in the criminal justice system is maintained. Level 3 may also be necessary when there is a perceived need to commit significant resources at short notice (Box 5).

\section{Practicalities of working with MAPPA}

\section{Identification of MAPPA offenders}

For most psychiatrists, any involvement with MAPPA will be about patients who are convicted offenders in contact with mental health services. Most of these cases will be classified as level 1 , where the patient has been assessed as being low or medium risk. In these cases, the mental health trust acts as the lead agency managing the risk process as with any other psychiatric patient, without the active involvement of other agencies.

For a mentally disordered offender to qualify for management under MAPPA he or she must be convicted of a sexual or violent offence as listed in schedule 15 of the Criminal Justice Act 2003 and sentenced to a hospital order under section 37 or 37/41 of the Mental Health Act 1983 (MHA), or a prisoner whose detention in hospital was directed by the sentencing court (section $45 \mathrm{~A}$ of the MHA) 
or by the Secretary of State (section 47 of the MHA). All MAPPA-eligible offenders should be identified within 3 days of sentence or admission to hospital.

Other offenders who have a past conviction for a violent or sexual offence and are detained under section 3 of the MHA or a 'notional section 37' of the MHA at the end of a prison sentence may qualify for MAPPA management if they continue to pose a significant risk of harm to others that would require management at MAPPA level 2 or 3 .

\section{Notification to MAPPA}

When a patient has been identified as eligible for MAPPA, a formal notification to the relevant MAPPA coordinator for the local area should be made. Notification does not constitute a formal referral, but it ensures that MAPPA subjects are identified before discharge and provides the opportunity both to share clinical information with the criminal justice agencies. Psychiatrists are advised to identify potential MAPPA-eligible patients early in their admission so that subsequent decisions about information sharing can be made in a timely fashion.

The Ministry of Justice (2012) advocates the routine notification and information sharing on all MAPPA-eligible mentally disordered offenders at designated points in their care pathway. For detained patients on restricted hospital orders this includes any planned move of the patient outside the secure perimeter (such as leave or transfer to another hospital) and also at the first care programme approach (CPA) meeting at which a discharge is considered. MAPPA guidance recommends that notification (for level 1) and/or referral (for levels 2 or 3 ) should be made at the point of first (usually unescorted) leave, but most importantly when discharge plans are being made, so that the MAPPA coordinator in the discharge locality area will be informed and can plan and contribute to risk management as necessary. Where forensic patients are in regional units away from their home area, initial leave may be in a different MAPPA locality from the final discharge area, and therefore notifications should be made to both the home and the host MAPPA areas.

However, there is no requirement in the Ministry of Justice MAPPA guidance to inform MAPPA about every single episode of leave. Routine notifications about every leave trip or variation in leave arrangements are unworkable and may force the clinician into an unhelpful and counterproductive monitoring role, which could interfere with a critical therapeutic alliance and increase, rather than decrease, the patient's risk. MAPPA patients on planned escorted home leave may receive unexpected visits by the police, which may be experienced as intrusive and disrupt the treatment process.

\section{Referral to MAPPA}

Formal referral of a MAPPA-eligible patient is only necessary if active multi-agency management, i.e. level 2 or 3 , is required. If the case is to be managed at level 1, referral is not necessary and notification alone is sufficient. The level of MAPPA involvement should be discussed when planning discharge at the patient's CPA meeting. If it is decided that the patient needs level 2 or 3 management, a designated member of the care team should complete the referral form and send it to the relevant MAPPA coordinator to convene a level 2 or 3 meeting. Such a meeting enables information sharing, the preparation of an inter-agency risk management plan and access to specialist resources.

The National Probation Service offender managers retain responsibility for MAPPA-eligible offenders transferred to hospital and should ensure that appropriate MAPPA notifications and referrals are made. As such they should be invited to care planning and pre-discharge meetings with mental health professionals.

\section{Liaison and attendance at MAPPA meetings}

For patients under the management of MAPPA at levels 2 or 3, their responsible clinician or another designated representative of the clinical team should be invited to attend MAPPA meetings in order to contribute to the discussion of individual cases. Attendance in person is the normal expectation, but if this is not possible, video/telephone conferencing or the provision of a written report or brief letter or email should be considered. Any information about the patient shared with other agencies should be the minimum necessary, and should not be disclosed by a MAPPA member without discussion or agreement of the MAPPA chair at the meeting.

In addition to the local mental health representative for individual MAPPA cases, each borough MAPPA (in London) or area MAPPA (outside London) has a permanent health representative, as required by the 'duty to cooperate'. The standing member may or may not have direct knowledge of the individual under discussion.

\section{Exit from MAPPA}

It is important to identify when it is no longer necessary for an offender to remain under MAPPA. The criteria for leaving MAPPA are different for each of its three categories of offender.

Category 1 offenders (registered sex offenders) may be discharged from MAPPA when their period of registration expires. Offenders whose MAPPA registration is for life are eligible to seek a 
review 15 years from the date of their first notification.

Category 2 offenders (violent offenders and other sexual offenders) are able to exit MAPPA when their licence expires, they are discharged from a hospital or guardianship order, or a disqualification order is revoked.

Category 3 offenders (other dangerous offenders) may exit MAPPA when a level 2 or 3 MAPPA meeting decides that the risk of harm has reduced sufficiently or the case no longer requires active multi-agency management.

\section{Confidentiality, information and disclosure}

The area that perhaps provokes the most confusion and disquiet regarding the relationship between psychiatry and MAPPA has been that of confidentiality and disclosure. Since MAPPA's inception, psychiatrists and others have warned against a shift in the role of the psychiatrist towards becoming an agent of the state as their efforts to protect the public from mentally ill patients can come at the expense of prioritising the patients' mental health and concomitant right to privacy. Moreover, as noted above, disclosure of information without the patient's knowledge or consent may threaten or destabilise an already fragile therapeutic relationship between the patient and their psychiatrist or keyworker.

\section{Information sharing and disclosure}

MAPPA guidance (Ministry of Justice 2012-revised in 2016) distinguishes between information sharing and disclosure. Information sharing is defined as the sharing of information among all of the agencies involved in MAPPA. Disclosure, on the other hand, is the sharing of specific information about a MAPPA offender with a third party (not involved in MAPPA) for the purpose of protecting the public. The third party could be a member of the public (such as a victim, an employer, a person forming a relationship with an offender) or a person acting in a professional capacity but not party to the MAPPA arrangements.

\section{The duty to cooperate versus maintaining confidentiality}

Although psychiatrists have a duty to cooperate with MAPPA, this does not extend to a statutory duty and does not mean an obligation to share or disclose information. Any information sharing or disclosure needs to be considered within the framework of professional guidance on confidentiality and disclosure. This includes guidance from the Royal College of Psychiatrists (2017), the General Medical Council (GMC) (2017) (Box 6),
BOX 6 General Medical Council guidance on MAPPA

In relation to MAPPA, the most recent GMC guidance on confidentiality states:

'You should consider the assessment of risk posed by patients made by other professionals and by groups established for that purpose, but you must make your own assessment and decision as to whether disclosure is justified. Your assessment of risk is a matter of professional judgement in which an offender's past behaviour will be a factor' (General Medical Council 2017: p. 72) the British Medical Association (no date) and the Department of Health (2003, 2010). The same medical duties of confidentiality and information governance apply as in normal clinical practice, and requests for information made by MAPPA agents should be treated as all other requests. Where psychiatrists are responsible for making information-sharing decisions it is still the clinical decision of the doctor to determine, on a case-bycase basis, whether disclosure is needed to prevent serious harm.

If information sharing is considered necessary, the information shared should be the minimum necessary to assist with risk assessment and management of individual cases and would rarely include handing over full psychiatric reports or medical records. It is also important to establish whether any disclosed information about the offender will be shared with individuals or agencies outside of MAPPA, as this will be relevant to the decision whether or not to share it in the first place.

\section{Disagreements regarding information sharing and disclosure}

Some mental health trusts are moving towards the implementation of procedures for the routine identification and notification to MAPPA of eligible cases. This raises concerns that information regarding these patients may be disclosed and shared without due consideration of the risks. Most decisions about MAPPA disclosure are made by consultant psychiatrists, particularly where they retain legal responsibility as responsible clinicians. However, in some cases other mental health professionals (such as social workers) may be involved in these decisions without medical oversight, which may result in information sharing that extends beyond the minimum needed. Such decisions may be open to scrutiny by patients and their representatives and lead to complaints and, in more serious cases, civil lawsuits.

Disagreements may also occur regarding the threshold above which disclosure of confidential information about a patient is deemed necessary. 
MCO answers

1 e 2 d 3 a 4 c $5 d$
Criminal justice agencies are likely to have a lower threshold than healthcare organisations, which may lead to tensions in working relationships between the MAPPA agencies. Tensions can also arise between mental health trusts and individual doctors regarding the information-sharing threshold. All trusts should have a trust policy on MAPPA as well as a designated MAPPA lead. Where there is disagreement within a multidisciplinary team as to whether or not information should be exchanged, the issue should be reviewed by a consultant colleague, clinical director and/or the Caldicott guardian. If members of the MAPPA team seek information and an individual mental health professional refuses, MAPPA chairs may refer the matter to the trust's chief executive, who carries the legal duty and can be the final arbitrator in such decisions.

What is perhaps more difficult to identify and address is the potential blurring of professional boundaries that may occur at MAPPA meetings where less experienced health representatives may be unprepared for the often subtle pressures placed on them to disclose information on individual patients. This highlights the importance of effective communication between mental health teams and the supervision of more junior colleagues, who may be unfamiliar with MAPPA and its interface with mental health practice, and the differing guidelines and regulations operating in criminal justice agencies and the NHS.

\section{Service user involvement}

MAPPA has been criticised for inadequate involvement of MAPPA-eligible patients or offenders in the MAPPA process. Although they should always be told of their MAPPA status, MAPPA patients or their representatives, including legal representatives, are excluded from MAPPA meetings. There is also no right of appeal against the decisions of a MAPPA meeting, although there are formal complaints procedures within the police and probation services. We are not aware of significant legal appeals or judicial reviews of MAPPA decisions. The situation of the MAPPA offender is therefore much more restricted than that of a patient detained under the Mental Health Act, with its inherent system of tribunals and appeals, and the common practice in mental healthcare where the patient is routinely invited to attend their regular CPA meetings.

The Criminal Justice Act 2003 does, however, stipulate that two lay advisors are appointed to each responsible authority area. This is a voluntary and unpaid job, and an advisor's role is to act as an informed observer, challenging the MAPPA professionals where appropriate as a 'critical friend', rather than participating in organisational decision-making.

\section{Conclusions}

The introduction of MAPPA can be viewed as arising from a move towards a 'blame' culture, as described by Douglas (1994), in which every bad outcome must be attributed to a failure or omission of a public official, with no acceptance of the 'slings and arrows of outrageous fortune'. The reason for increasing focus of attention by Western societies on sex offenders is unclear, given that in Dante's inferno people overcome by lust only occupied the second circle of hell.

However, MAPPA has now been existence for over 15 years and its evolution reflects a shift towards individual autonomy and a focus on the rights of vulnerable victims, as well as increasing societal and political demands for sharing information about risky individuals that would otherwise be confidential. Inquiries into homicides and other serious offences frequently highlight failures in communication between agencies that might have contributed to the offence, and clearly some degree of contact between the different professionals and services working with patients and offenders is essential to mitigate the risks they may pose. In our view, MAPPA is an acceptable and practical alternative to the blanket disclosure of information about sex offenders that has been called for in the British tabloid press.

Nevertheless, the primary duty of psychiatrists is to care for their patients, and any decision to violate their privacy by disclosing information about them without their consent must not be made lightly. The legal framework regarding confidentiality is not clear cut, and the guidelines and codes of conduct regarding disclosure and information sharing of different professional groups may differ or even contradict each other.

Current legislation, as outlined in the GMC guidance, still allows doctors the freedom to use their own clinical judgement in making any decision to disclose confidential details about a patient without their consent, without any mandatory duty to report. This includes requests for information under the duty-to-cooperate stipulation of MAPPA. At the same time, they have a duty to cooperate by working thoughtfully and respectively with MAPPA and the other agencies involved with mentally disordered offenders, not only to protect the public but in the best interests of their patients. Our own experience of working with MAPPA, both at local level and on one of its strategic management boards, has been for the most part positive in participating in thoughtful discussions about offenders, in which interest in and respect for the individual's history, current circumstances and lived experience are shown by the different agencies involved and contribute to decisionmaking. 


\section{References}

British Medical Association (no date) Confidentiality and Disclosure of Health Information Tool Kit. BMA (http://bma.org.uk/practical-supportat-work/ethics/confidentiality-tool-kit). Accessed 18 March 2018.

Department of Health (2003) Confidentiality: NHS Code of Practice. Department of Health.

Department of Health (2010) Supplementary Guidance on Public Interest Disclosures. Department of Health.

Douglas M (1994) Risk and Blame: Essays in Cultural Theory. Routledge.

Fitch K (2007) Sex offender management: children's rights, Megan's Law and the child sex offenders review. ChildRight, 238: 18-21.

General Medical Council (2017) Confidentiality. GMC Publications.
Joseph N, Benefield N (2012) A joint offender personality disorder pathway strategy: an outline summary. Criminal Behaviour \& Mental Health, 22: 210-17.

Liebling A, Crewe B (2012) Prison life, penal power and prison effects. In The Oxford Handbook of Criminology (5th edn) (eds M Maguire, R Morgan, R Reiner): 895-927. Oxford University Press.

Ministry of Justice (2012) MAPPA Guidance 2012, Version 4.1, Updated 2016.

Royal College of Psychiatrists (2017) Good Psychiatric Practice: Confidentiality and Information Sharing (3rd edn) (College Report CR209). Royal College of Psychiatrists.

Taylor R, Yakeley J (2013) Working with MAPPA: Guidance for Psychiatrists in England and Wales (Faculty Report FR/FP/01). Royal College of Psychiatrists.

\section{MCOs}

1 Regarding legislation related to MAPPA:

a following the implementation of 'Megan's Law' in the USA, more child sex offenders were identified than previously

b 'Sarah's Law' in the UK allows the public to find out details regarding sex offenders in their area

c the Sexual Offences Act 2003 bans MAPPA offenders from travelling abroad

d the Criminal Justice Act 2003 shortened the length of sentences for sexual offences

e the Domestic Violence, Crime and Victims Act 2004 allows victims of domestic abuse by MAPPA-eligible offenders to be told when the offender is being discharged from hospital.

2 The duty to cooperate:

a requires all MAPPA agencies to share information regarding every MAPPA-eligible offender

b gives MAPPA the overall responsibility for each agency's decision to disclose information about the offender

c requires the offender's individual mental health representative to be responsible for any decisions made regarding the offender's mental health

d allows the different agencies to make decisions regarding information sharing and disclosure in line with their own codes of conduct and professional regulations e mandates disclosure of personal information regarding the offender in cases where that individual has been assessed as posing a very high risk to others.

3 In the MAPPA framework:

a the establishment of the core functions of MAPPA (identification of offenders, assessment and management of risk, and sharing of information) is the responsibility of the responsible authority

b MAPPA categories reflect the type of offence and psychiatric diagnosis of the offender

c decisions regarding level 2 offenders always require input from the MAPPA mental health representative

d MAPPA level 2 pertains only to sexual offenders

e MAPPA level 3 is solely for high-risk offenders.

\section{In working with MAPPA:}

a once a patient is identified as being eligible for MAPPA they must be referred immediately

b referral to MAPPA is necessary for all three levels of management

c for MAPPA-eligible offenders in prison, the National Probation Service is responsible for ensuring MAPPA notifications and referrals d for any MAPPA-eligible patient detained under the Mental Health Act, all leave arrangements must be reported to the Ministry of Justice

e category 3 offenders remain under MAPPA for life.

5 Regarding confidentiality, information and disclosure:

a disclosure is the sharing of information between all of the MAPPA agencies involved in the case and information sharing is the sharing of specific information about a MAPPA offender with a third party

b in the UK disclosure about very serious offences that are likely to cause harm to the public is mandatory

c each duty-to-cooperate agency must abide by the requirements for disclosure set by MAPPA

d psychiatrists may decide not to disclose information regarding an offender despite being asked to do so by the MAPPA responsible authority

e offenders must be informed of every decision made about them by MAPPA. 